\title{
Are We Beyond Cowboy Tactics for Arterial Catheterization?
}

Ultrasound guidance for vascular cannulation has been proposed to increase success rates for vascular access and decrease complications. ${ }^{1,2}$ Ultrasound is also a tool for choosing the optimal target vessel: identifying size, position, and patency is especially important under certain conditions (peripheral vascular disease, obesity, hypotension). However, studies vary by methodology, patient population, operator population, and clinical setting. The routine use of ultrasound guidance requires not only additional training and experience of the operator but also equipment availability in both elective and emergency settings.

\section{See the Original Study on Page 1813}

The figure shows the left arm of an 82-year-old patient with chronic hypertension and severe aortic stenosis after multiple unsuccessful attempts by an experienced cardiovascular anesthesiologist to place an arterial line without ultrasound guidance. Both arms had similar hematoma formation, and the patient remembers the event as lengthy and very painful. Although not a "never event" or a serious reportable event, hematoma is a largely preventable complication of peripheral arterial catheterization. ${ }^{3}$ With current evidence suggesting that ultrasound-guided radial artery line placement results in a higher first-attempt success rate and fewer complications, this hematoma was likely preventable. ${ }^{2}$ So why wasn't ultrasound guidance used?

Some experienced health-care professionals are known for taking a cowboy approach to procedures; that is, they prefer to perform skills in a manner that other people might consider imprudent or even dangerous. The ego of a cowboy often drives the dismissal of published guidelines or recommendations, and thus the I've-always-doneit-this-way approach to care remains highly prevalent. In this case of an arterial catheterization attempt resulting in bilateral massive hematomas, a cowboy might defend such an outcome as a known complication.

Dr Benedik has disclosed no conflicts of interest.

Correspondence: Penelope S Benedik PhD CRNA RRT-NPS, UTHSCHouston, Nurse Anesthesia, 6901 Bertner Avenue, \#682, Houston, TX 77030. E-mail: psvillars@gmail.com.

DOI: $10.4187 /$ respcare 03800

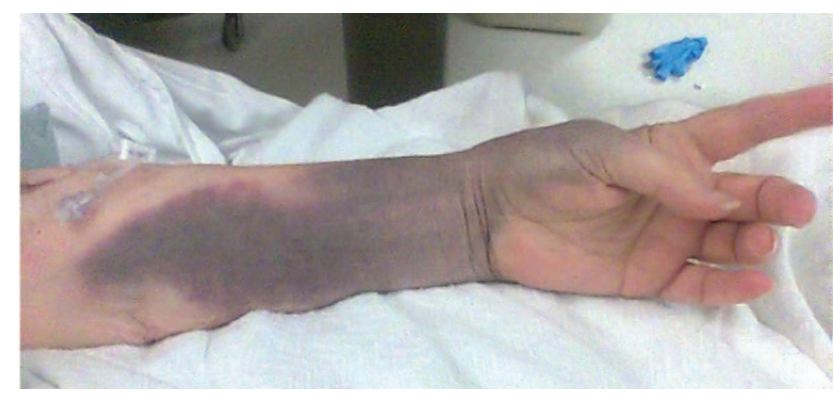

Arm of patient after multiple attempts at arterial catheterization.

Is hematoma as a result of arterial cannulation simply a known complication, or is it an adverse event or even an error? A complication is an unanticipated problem that arises following, and is a result of, a procedure, treatment, or illness. ${ }^{4} \mathrm{~A}$ hematoma after multiple arterial punctures can be anticipated, therefore by definition it is more than a complication. Additionally, a complication is generally characterized as the unfavorable evolution of a disease process or condition, whereas an intervention such as a drug or treatment that causes harm is usually characterized as an adverse event. An adverse event is an injury or harm resulting from medical care. This seems a more appropriate categorization of such a hematoma.

Patient safety literature now differentiates between a preventable adverse event and a nonpreventable adverse event, and this hematoma generally falls into the first category. ${ }^{5}$ An error is "an act of commission (doing something wrong) or omission (failing to do the right thing) leading to an undesirable outcome." "Could failure to utilize ultrasound guidance for arterial cannulation be considered an error of omission or even an error at the sharp end in which the event is readily apparent and committed by the provider directly caring for the patient? If a failure to use ultrasound guidance is an error, is it a slip (due to lack of concentration) or a mistake (failure of conscious thought, analysis, and planning)? According to Reason, ${ }^{7}$ mistakes are the primary type of error in the planning stage of cognition. In this case, the risk of difficult arterial catheterization was high due to the patient's age and comorbidities. Was the omission of ultrasound guidance due to a cowboy attitude? Should it be classified as a mistake, or 


\section{Are We Beyond Cowboy Tactics for Arterial Catheterization?}

shall we call it poor clinical judgment? It is unlikely that there will be consensus on the answers to these questions.

Responsibility for this type of outcome cannot be placed solely on the individual health-care provider. Other questions emerge, such as are providers given the time and resources to evaluate emerging new evidence? Is there a department-level commitment to applying new findings to clinical practice? Is the department's culture one that allows for the application of a new approach, or would the provider have been ridiculed? Does the institution provide appropriate training and experience in the use of ultrasound? Is the equipment available to support the provider's choice to use it?

In a recent review on barriers to evidence-based medicine, multiple contributory factors were identified. Sadeghi et $\mathrm{al}^{8}$ reported that among the subgroup of hospital or specialist care providers, the 5 most common barriers to implementing evidence-based practice were (1) lack of time, (2) research barriers, (3) lack of resources, (4) inadequate skills, and (5) cooperate barriers. Lack of time included inadequate or no time given to search for and study the available evidence and insufficient time provided to organize and implement the findings. Research barriers included lack of understanding research methodologies, statistical analyses, and/or the clinical relevance of the reports. A lack of resources is a common barrier to implementation of guidelines, as equipment, supplies, and institutional support are not always forthcoming. The last barrier speaks to the culture of a setting, in which there is a lack of teamwork or unwillingness to cooperate with change.

What evidence is available on this issue? The most recent meta-analysis of the efficacy of ultrasound-guided arterial catheterization reported that ultrasound guidance was associated with an increased first-attempt success rate compared with palpation (relative risk of $1.55,95 \%$ CI $1.02-2.35, P=.04)$, along with reduced mean attempts to access (weighted mean difference of $-1.13,95 \% \mathrm{CI}-1.58$ to $-0.69, P<.001$ ), reduced mean time to access (weighted mean difference of $-72.97 \mathrm{~s}, 95 \% \mathrm{CI}-134.41$ to -11.52 , $P=.02$ ), and lower risk of hematoma (relative risk of $0.17,95 \%$ CI $0.07-0.41, P<.001) .^{2} \mathrm{Gu}$ et al ${ }^{2}$ analyzed both adult and pediatric populations $(n=546)$, emergency and elective settings, and operators from several specialties (anesthetists, emergency specialists) with varying degrees of experience. Thus, there is compelling evidence that the use of ultrasound guidance for arterial catheterization is effective and beneficial, and reduces complications.

Additional related studies have been published that provide equivocal support for these findings. In a prospective study of 183 adult subjects scheduled for cardiac catheterization, Zaremski et $\mathrm{al}^{9}$ found no differences in firstattempt success rates between ultrasound-guided versus palpation radial artery access $(87 \%$ vs $86.8 \%, P=.99)$ and in access time (47 s [interquartile range of 20-90 s] vs $31 \mathrm{~s}$ [interquartile range of $20-75 \mathrm{~s}$ ], $P=.18$ ). Notably, in this study, the operators were experienced interventional cardiologists.

Bobbia et $\mathrm{al}^{10}$ reported a randomized controlled trial of 72 adult subjects who presented to the emergency room and needed arterial blood sampling (not catheterization). The operators were both novice and experienced emergency room physicians, all of whom had previous training in ultrasound that was refreshed with simulator training before the study. There was no difference in the number of attempts to successfully draw the arterial blood sample (2.35 vs $1.66, P=.17$ ), and the time to retrieve the sample was higher in the ultrasound group compared with the palpation group (132 vs $55 \mathrm{~s}, P<.01)$.

More data on the efficacy of ultrasound guidance may be culled from quality-improvement projects. In a recent prospective study by Varkey and Dumitru, ${ }^{11}$ ultrasound guidance was compared with palpation for placement of radial artery catheters in subjects scheduled for cardiovascular surgery $(n=80)$. Operators in this project included certified registered nurse anesthetists, nurse anesthesia residents, staff anesthesiologists, and medical anesthesia residents; the trainees received instruction on using ultrasound before participating in the study. Ultrasound guidance compared with the palpation technique resulted in higher first-attempt success rates ( $85 \%$ vs $60 \%$ ) and fewer hematomas $(2.5 \%$ vs $17.5 \%)$.

In this edition of Respiratory Care, Miller et al ${ }^{12}$ contribute to this body of work and report the outcome of a program in which respiratory therapists with pre-existing experience in arterial catheterization were trained to use an ultrasound-guided technique. One-hundred twenty-two subjects who presented for emergency care participated; the use of ultrasound was discretionary for the respiratory therapist, and data were collected prospectively. The firstattempt success rate was $63.1 \%$, and the final success rate was $86.1 \%$ compared with historical success rates with the palpation technique of $77.8 \%$ at this institution. These data compare favorably with the most recent meta-analysis by $\mathrm{Gu}$ et al, ${ }^{2}$ who reported first-attempt success rates of $48.5 \%$ and $30.7 \%$ in the ultrasound and control groups, respectively.

Experts from the International Consensus Conference on Ultrasound Vascular Access formulated a set of evidence-based recommendations that was published in $2012 .{ }^{13}$ The panel of specialists reviewed 229 articles and formulated 47 evidence-based recommendations. The salient recommendation for ultrasound vascular access in adults was based on the level of evidence (A, high quality), the degree of consensus (very good), and the strength of the recommendation (strong): "Ultrasound-guided arte- 


\section{Are We Beyond Cowboy Tactics for Arterial Catheterization?}

rial catheterization improves first-pass success and should be used routinely in adults." 13

Thus, collective data from various patient populations with a variety of trained operators in diverse settings and current evidence-based recommendations may serve to challenge the cowboy's experiential approach to arterial vascular access. It is possible that ten years from now, we will look back on an era of cowboy tactics for arterial vascular access in awe. There was a time when I drew arterial blood gases with my bare hands using a manually heparinized glass syringe after a quick alcohol swipe. Times have clearly changed, and at some point, health-care providers must change with them.

Penelope S Benedik PhD CRNA RRT-NPS Nurse Anesthesia Division University of Texas Health Science Center at Houston Houston, Texas

\section{REFERENCES}

1. Egan G, Healy D, O’Neill H, Clarke-Moloney M, Grace PA, Walsh SR. Ultrasound guidance for difficult peripheral venous access: systematic review and meta-analysis. Emerg Med J 2013;30(7):521526.

2. Gu WJ, Tie HT, Liu JC, Zeng XT. Efficacy of ultrasound-guided radial artery catheterization: a systematic review and meta-analysis of randomized controlled trials. Crit Care 2014;18(3):R93
3. Agency for Healthcare Research and Quality. Patient safety primers. http://psnet.ahrq.gov/primer.aspx?primerID=3. Accessed September 27, 2014

4. http://medical-dictionary.thefreedictionary.com/Complications. Accessed September 24, 2014

5. Wachter RM. Understanding patient safety. New York: McGrawHill Medical; 2008.

6. Agency for Healthcare Research and Quality. Glossary. http://psnet. ahrq.gov/glossary.aspx. Accessed September 27, 2014.

7. Reason J. Human error. Cambridge, England: Cambridge University Press; 1990.

8. Sadeghi-Bazargani H, Tabrizi JS, Azami-Aghdash S. Barriers to evidence-based medicine: a systematic review. J Eval Clin Pract 2014 [Epub ahead of print] doi: 10.1111/jep.12222

9. Zaremski L, Quesada R, Kovacs M, Schernthaner M, Uthoff H. Prospective comparison of palpation versus ultrasound-guided radial access for cardiac catheterization. J Invasive Cardiol 2013;25(10): 538-542.

10. Bobbia X, Grandpierre RG, Claret PG, Moreau A, Pommet S, Bonnec $\mathrm{JM}$, et al. Ultrasound guidance for radial arterial puncture: a randomized controlled trial. Am J Emerg Med 2013;31(5):810-815.

11. Varkey A, Dumitru R. Ultrasound-guided arterial line procurement. Practice Inquiry Project. Houston, Texas: University of Texas Health Science Center at Houston; 2014.

12. Miller AG, Cappiello JL, Gentile MA, Almond AM, Thalman JJ, MacIntyre NR. Analysis of radial artery catheter placement by respiratory therapists using ultrasound guidance. Respir Care 2014; 59(12):1813-1816.

13. Lamperti M, Bodenham AR, Pittiruti M, Blaivas M, Augoustides JG, Elbarbary $\mathrm{M}$, et al. International evidence-based recommendations on ultrasound-guided vascular access. Intensive Care Med 2012; 38(7):1105-1117. 